\title{
A retrospective dosimetry study of intensity-modulated radiotherapy for nasopharyngeal carcinoma: radiation- induced brainstem injury and dose-volume analysis
}

Cheng-Yun Yao ${ }^{1+}$, Guo-Ren Zhou ${ }^{2+}$, Li-Jun Wang ${ }^{1}$, Jian-Hua Xu' ${ }^{1}$ Jin-jun Ye ${ }^{1}$, Lan-Fang Zhang ${ }^{3}$, Xia He ${ }^{1}$, Zhen-Zhang Chen ${ }^{1 *+}$ and Sheng-Fu Huang ${ }^{1 *+}$ (D)

\begin{abstract}
Background: Radiation therapy is the standard radical treatment for nasopharyngeal carcinoma (NPC) but also causes transient as well as long-term complications. Patients who develop severe radiation-induced brainstem injuries have a poor prognosis due to the lack of effective medical therapies. However, the relationship between brainstem injury and radiation volume dose is unknown. In this study, we found that radiation-induced brainstem injury was significantly associated with brainstem dose per unit volume.

Methods: A retrospective analysis was performed on a consecutive cohort of 327 patients with NPC receiving IMRT from May 2005 to December 2014. Dose-volume data and long-term outcome were analyzed.

Results: The median follow-up duration was 56 months (range, 3-141 months), and six with $T_{4}$ and two with $T_{3}$ patients had radiation-induced brainstem injuries. The 3-year and 5-year incidences were $2.2 \%$ and $2.8 \%$, respectively. The latency period of brainstem injury ranged from 9 to 58 months, with a median period of 21 months. The Cox regression analysis showed that brainstem radiation toxicity was associated with the $T_{4}$ stage, $D_{2 \%}$ of gross tumor volume of nasopharyngeal primary lesions and their direct extensions (GTVnx), $D_{\max }$ (the maximum point dose), $D_{1 \%}$ $\mathrm{D}_{0.1 \mathrm{cc}}$ (the top dose delivered to a $0.1-\mathrm{ml}$ volume), and $\mathrm{D}_{1 \mathrm{cc}}$ (the top dose delivered to a 1-ml volume) of the brainstem $(p<0.05)$. Receiver operating characteristic (ROC) curves showed that $G T V n x D_{2 \%}$ and the $D_{\max } D_{1 \%}, D_{0.1 c c}$ and $D_{1 c c}$ of the brainstem were significant predictors of brainstem injury. The area under the ROC curve for these five parameters was $0.724,0.813,0.818,0.818$, and 0.798 , respectively $(p<0.001)$, and the cutoff points $77.26 \mathrm{~Gy}, 67.85 \mathrm{~Gy}, 60.13 \mathrm{~Gy}$, $60.75 \mathrm{~Gy}$, and $54.58 \mathrm{~Gy}$, respectively, were deemed as the radiation dose limit.
\end{abstract}

Conclusions: Radiotherapy-induced brainstem injury was uncommon in patients with NPC who received definitive IMRT. Multiple dose-volume data may be the dose tolerance of radiation-induced brainstem injury.

Keywords: Nasopharyngeal carcinoma, Intensity-modulated radiotherapy, Brainstem, Radiation injury

\footnotetext{
*Correspondence: zhenzhangchen@163.com; huangsf2009@163.com

${ }^{\dagger}$ Cheng-Yun Yao, Guo-Ren Zhou, Zhen-Zhang Chen and Sheng-Fu Huang contributed equally to this work.

${ }^{1}$ Department of Radiation Oncology, Jiangsu Cancer Hospital \& Jiangsu Institute of Cancer Research \& The Affiliated Cancer Hospital of Nanjing Medical University, 42 Baiziting Road, Nanjing 210009, People's Republic of China

Full list of author information is available at the end of the article
}

(c) The Author(s). 2018 Open Access This article is distributed under the terms of the Creative Commons Attribution 4.0 International License (http://creativecommons.org/licenses/by/4.0/), which permits unrestricted use, distribution, and reproduction in any medium, provided you give appropriate credit to the original author(s) and the source, provide a link to the Creative Commons license, and indicate if changes were made. The Creative Commons Public Domain Dedication waiver (http://creativecommons.org/publicdomain/zero/1.0/) applies to the data made available in this article, unless otherwise stated. 


\section{Background}

Nasopharyngeal carcinoma (NPC) is rather common among Asians, especially the Southern Chinese [1]. Radiation therapy is the standard radical treatment for NPC but also causes transient as well as long-term complications [2]. Radiation-induced brain necrosis ( $\mathrm{RN}$ ) is one of the more severe complications and can potentially lead to cognitive dysfunction, seizure, headache, and limb paralysis. The incidence of RN has been demonstrated to directly correlate with the modality of radiation therapy, which was suggested in previous studies; there is a lower occurrence in patients treated with intensity-modulated radiotherapy (IMRT) [3]. However, there are other factors that may influence the incidence and severity of radiation-induced brainstem injury, for example, long-term close follow-up, the proper diagnostic modality, and independent image interpretation.

The authors of this study recognized the importance of those aspects and accordingly analyzed the brainstem data of NPC patients treated in our center between May 2005 and December 2014. We also try to identify the relationship between the incidence of brainstem injury and radiation dose to improve the understanding of brainstem protection.

\section{Methods}

\section{Patients}

Inclusion criteria were as follows: (1) histologically confirmed NPC by biopsy; (2) no evidence of distant metastasis; (3) no previous treatment for NPC; (4) no pregnancy or lactation; (5) no previous malignancy or other concomitant malignant disease; (6) performance status of 0 or 1 ; (7) received radical IMRT at initial diagnosis; (8) no brain bleeding history; and(9)regular close follow-up with contrasted MRI. From March 2005 to September 2014, 327 newly diagnosed, biopsy-proven, consecutive NPC patients were treated in Jiangsu Cancer Hospital. 19 patients (5.5\%) did not meet the research requirements and were excluded from the study. All participants were provided their written informed treatment consent, the development of this retrospective study has been approved by hospital ethics committee and is in line with the Helsinki Declaration. and all experiments were performed in accordance with relevant guidelines and regulations. Of these, magnetic resonance imaging (MRI) data allowing brainstem evaluation after completion of IMRT were available for 327. The male/female ratio was 2.7:1, and patients ranged from 12 to 77 years old (median, 48 years old). According to the 7 th edition of the AJCC/UICC staging system, 24 patients had stageIdisease, 59 stage II, 111 stage III, and 133 stage IVa. By T-stage classification, 102 patients were T1, $32 \mathrm{~T} 2$, $80 \mathrm{~T} 3$, and $113 \mathrm{~T} 4$. All patients underwent a series of pretreatment evaluations and examinations (including history-taking, physical examination, hematological and biochemical profiling, nasopharynx and neck contrasted MRI, thoracic-abdominal computed tomography (CT), and whole- body single photon emission CT bone scanning) to exclude those with contraindications to treatment and distant metastases. This retrospective study was approved by the ethics committee of Nanjing Medical University Cancer Center.

\section{IMRT and chemotherapy}

Inverse IMRT treatment planning was performed on a Varian Inspiration Platform (version 10.0), using the simultaneous integrated boost technique. Gross tumor volume of nasopharyngeal primary lesions and their direct extensions (GTVnx) and positive neck lymph nodes (GTVnd) were delineated according to the recommendations of the International Commission on Radiation Units and Measurements Reports nos. 50 and 62. The clinical target volume 1 (CTV1) was defined as the GTVnx with 5-10-mm margins to encompass areas at high risk of microscopic extension and the entire nasopharyngeal mucosa plus a 5-mm depth of sub-mucosal tissue. The CTV2 was defined by addition of $3-10-\mathrm{mm}$ margins to the CTV1 to include areas at low risk of microscopic extension, the level of the identified positive lymph node, and the elective cervical region. The corresponding planning target volumes (PTVs) were generated from the GTVs or CTVs plus 3-mm margins to allow for setup uncertainties. The prescribed doses were 68-75 Gy to the PTV of the GTVnx in 32-34 fractions; 64-75 Gy to the PTV of the GTVnd in 32-34 fractions; 60 Gy to the PTV of CTV1 in 32 fractions; and 50 Gy to the PTV of CTV2 in 28 fractions. All patients were given one fraction daily 5 days a week. The dose-volume-histograms (DVHs) of the organs at risk were evaluated as described in the radiation therapy oncology group (RTOG) 0225 protocol to prevent violation of the tolerance limits [4].

IMRT alone was recommended for stageIpatients and IMRT combined with concurrent platinum-based chemotherapy for stage II-IVb patients [5]. Neoadjuvant chemotherapy was prescribed for patients with bulky lesions (at the primary site or in the neck); those with residual disease after IMRT received platinum-based adjuvant chemotherapy.

\section{Brainstem re-contouring and DVH data collection}

As the brainstem had been delineated inconsistently by different radiation oncologists during original IMRT planning, we used a recommended method [6] to re-contour the brainstem. This allowed us to collect accurate data on the following dosimetric parameters: the mean dose $\left(D_{\text {mean }}\right)$, the maximum point dose $\left(D_{\max }\right)$, $\mathrm{D}_{1 \%}, \mathrm{D}_{0.1 \mathrm{cc}}$ (the maximum dose delivered to a volume of 
$0.1 \mathrm{ml}$; the following seven parameters are similar), $\mathrm{D}_{1 \mathrm{cc}}$, $\mathrm{D}_{5 \mathrm{cc}}, \mathrm{D}_{10 \mathrm{cc}}, \mathrm{D}_{15 \mathrm{cc}}, \mathrm{D}_{20 \mathrm{cc}}, \mathrm{D}_{25 \mathrm{cc}}$, and $\mathrm{D}_{30 \mathrm{cc}}$. In addition, clinical variables, such as age, sex, stage, $\mathrm{GTV}_{\mathrm{nx}}$, and chemotherapy use, were included in this study; the anteroposterior diameter of the pons cistern was also analyzed for brainstem injury.

\section{Image assessment and the criteria for diagnosis and grading of radiation brainstem injury}

The endpoint of analysis was the development of brainstem injury identified by MRI after irradiation. All MR images were reviewed independently by two examiners (L.F.Z. and S.F.H.) who were specialized in head-and-neck cancer. Consensus was reached by discussion if any initial disagreement was apparent. As both Quality-of-life (QoL) and brain function were of great importance, regular close assessment of brainstem function was always conducted after imaging diagnosis.

Diagnostic criteria for brainstem radiation toxicity refer to RN diagnosis. It is defined as a lesion of high signal on T2-weighted images and a lesion of enhancement on post-contrast images, particularly with "soap bubble" or "Swiss cheese" enhancement [7, 8]. All brainstem injury was graded according to the Common Terminology Criteria for Adverse Events (CTCAE) version 4.03, which was based on the clinical symptoms (Grade 1-mild or asymptomatic; Grade 2-moderate, not interfering with activities of daily living (ADLs); Grade 3-severe interference with ADLs, possible intervention; Grade 4-life-threatening or disabling, intervention indicated; and Grade 5-death).

\section{Follow-up and statistical methods}

Follow-up included clinical assessment and MRI evaluation of the head and neck. The follow-up duration was calculated from the end of IMRT to the day of the final scan. All patients were regularly followed up every 3 months during the first year, every 3-6 months during the next 2 years, and annually thereafter. The use of MRI examination on the head and neck during follow-up was conducted well, and more close MRI evaluation of the nasopharynx and/or neck was performed for cases with suspected tumor recurrence or radiotherapy-induced complications. A total of 2943 MR images were collected during follow-up; an average of approximately nine scans were available for each patient. The latency period of radiotherapy-induced brainstem complications was measured from the time of IMRT completion to the first appearance of brainstem injury.

All statistical analyses were performed using SPSS software version 21.0 (SPSS, Chicago, IL, USA). Significant dosimetric parameters were further tested using the Cox proportional hazards model. Independent significant factors were assessed using receiver operating characteristic
(ROC) curves to estimate the brainstem dose tolerance. Two-sided $p$-values $\leq 0.05$ were considered statistically significant.

\section{Results}

Survival and patterns of treatment failure

The median follow-up duration was 56 months (range, 3-141 months). The 3-year, 5-year, overall survival, local control rate, and free from distant metastasis survival rate were $88.5 \%, 78.7 \%, 93.4 \%, 91.4 \%, 85.0 \%$, and $81.4 \%$, respectively. The median time to recurrence was 54 months (range, 3-141 months), and that to development of distant metastasis was 52 months (range, 2141 months). Seventy (21.4\%) patients died during the follow-up period.

\section{Incidence and latency period of brainstem injury}

A total of 8 cases (8/327) developed MRI-indicated radiotherapy-induced brainstem injury. The actuarial incidence rates were $2.2 \%$ and $2.8 \%$ at 3 and 5 years, respectively; and these were $2.5 \%$ and $5.3 \%$ in $\mathrm{T} 3$ and T4 patients, respectively (Fig. 1). The median latency period was 21 months (range, 9-58 months).

\section{Clinical manifestations and characteristics of MRI}

Of the eight patients who developed brainstem damage, four patients exhibited varying degrees of clinical symptoms, including lower cranial nerve palsy symptoms and fatigue; one case developed progressive limb weakness, finally becoming hemiplegic; and one patient died, with personality changes before death. The remaining 4 patients were asymptomatic. All brainstem injury patients

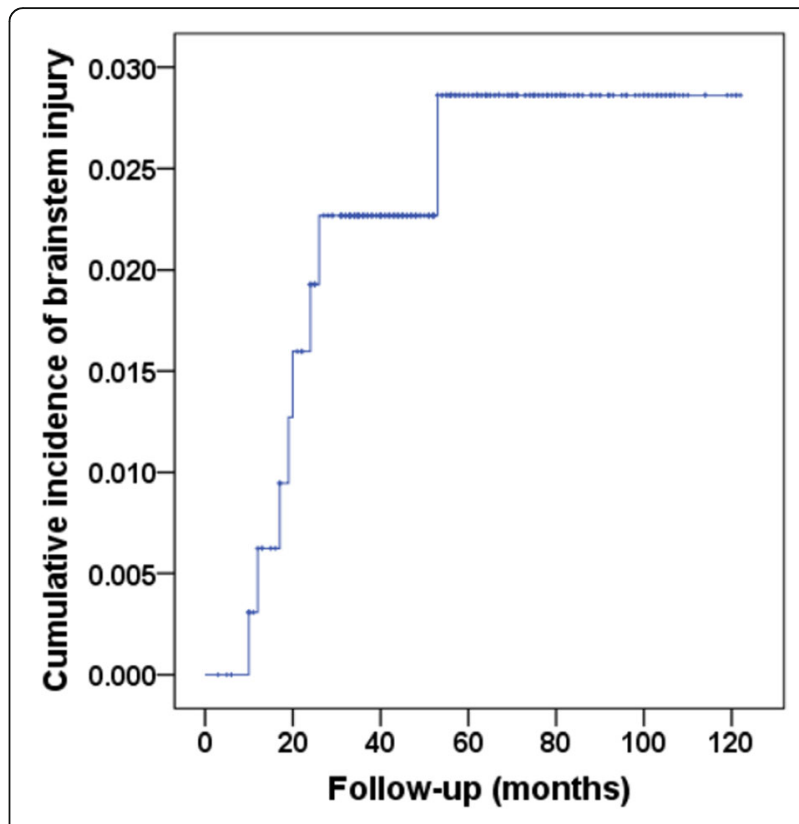

Fig. 1 Cumulative incidence of radiation-induced brainstem injury 
were of stage $\mathrm{T}_{3}$ ( 2 cases) or $\mathrm{T}_{4}$ (6 cases) and received chemotherapy during their treatment periods. The corresponding dose distributions are shown in Table 1.

Overall, the MRI findings in the brainstem injury patients revealed a continuous spectrum of RT-associated damage. Small solid enhanced nodules were evident in four cases, while moderate and large lesions were apparent in other cases (lesions were classified as described in Table 1). The evolution of radiotherapy-induced brainstem injury was well documented; the MRI features of brainstem injury (including signal abnormality on $\mathrm{T} 1$ and T2 images) resolved completely in three patients following gradual improvement over two months. However, one patient developed a large contrast-enhanced lesion, with a central necrotic core in the base of the pons; this was the only patient with brainstem injury who died (Fig. 2).

\section{Predictive factors of radiation brainstem injury}

The $\mathrm{T}$ category $\left(\mathrm{T}_{4}\right.$ vs $\left.\mathrm{T}_{1-3}\right)$ as well as GTVnx $\mathrm{D}_{2 \%}$, $\mathrm{D}_{\text {max }}, \mathrm{D}_{1 \%}, \mathrm{D}_{0.1 \mathrm{cc}}$, and $\mathrm{D}_{1 \mathrm{cc}}$ of the brainstem were predictive factors of the radiation-induced brainstem injury in Cox regression models $(p<0.05)$ (Table 2). However, year, sex, N stage, GTVnx $D_{98 \%}$, chemotherapy, anteroposterior diameter of the pons cistern, and $\mathrm{D}_{\text {mean }}$ were not independent risk factors of radiation-induced brainstem injury.

Five qualitative variables of the predictive factors were also demonstrated by ROC curves for brainstem injury (area under the ROC curves, Table 3); the cutoff points for the dose tolerance for brainstem injury for each parameter were selected using $P<0.05$ and Youden's index. The parameters and cutoff values are shown in Table 4 . A cumulative DVH for the dose tolerance of brainstem injury was drawn using the cutoff values (Fig. 3). The curves showed an increasing probability of brainstem injury with increasing dose; based on Fig. 3, it would be appropriate to propose a GTVnx $\mathrm{D}_{2 \%}$ of $77.26 \mathrm{~Gy}, \mathrm{D}_{\max }$ of $67.85 \mathrm{~Gy}$,
$\mathrm{D}_{1 \%}$ of $60.13 \mathrm{~Gy}, \mathrm{D}_{0.1 \mathrm{cc}}$ of $60.75 \mathrm{~Gy}$, and $\mathrm{D}_{1 \mathrm{cc}}$ of $54.58 \mathrm{~Gy}$ as the cutoff values for radiation-induced brainstem injury.

\section{Discussion}

Although radiation-induced brainstem injury is uncommon, its severity has received increasing attention. Brainstem injury patients may exhibit III-XII cranial nerve palsy as well as long-beam (cone and sensory system) and cerebellar injury symptoms. Patients have no clinical symptoms in mild cases; serious complaints vary and include limb weakness, hemiplegia, gait instability, temperature sensory disturbance, diplopia, dysarthria, tongue and facial paralysis, etc. [9]. Some patients may recover from the disease after their brainstem suffers mild radiation injury, while others may need earlier medical intervention to alleviate their symptoms. However, patients who developed severe radiation brainstem injuries have a poor prognosis due to the lack of effective medical therapies.

In our research, 193 patients (59.1\%) were classified as stage $\mathrm{T}_{3}$ or $\mathrm{T}_{4}$, and $8(2.4 \%)$ brainstem injuries occurred. Patients with brainstem injuries were stage $\mathrm{T}_{3}$ or $\mathrm{T}_{4}$ of the disease, which might have resulted from the high dose of RT to the brainstem because of large tumor invasion to the skull base or to the intracranium. Both studies $[10,11]$ showed that brainstem injuries are related to targets that are larger and closer to the brainstem, which is similar to our results, but there was no statistical difference between patients with $T_{3}$ and other cases, which may be due to the low rate of brainstem injury. Moreover, lack of MRI-based planning [11], the number of surgeries, hydrocephalus, diabetes, and hypertension [11-13] also contributed to injury of the brainstem. The pons cistern is located between the brainstem and basilar clivus; its anteroposterior diameter may have an impact on the brainstem dose. Our research demonstrated that there was a great variation

Table 1 Characteristics of 8 patients with brainstem injury

\begin{tabular}{|c|c|c|c|c|c|c|c|c|}
\hline \multirow[t]{2}{*}{ patient } & \multirow[t]{2}{*}{ gender } & \multirow[t]{2}{*}{ age } & \multirow[t]{2}{*}{ stage } & \multirow[t]{2}{*}{ Volume of GTVnx $\left(\mathrm{cm}^{3}\right)$} & \multicolumn{4}{|l|}{ Lesion } \\
\hline & & & & & Site & Number & size & grade \\
\hline 1 & male & 59 & T4NOMO & 164.6 & Junctional portion of pons-oblongata & 1 & $0.5 \times 0.7 \times 0.8$ & 1 \\
\hline 2 & male & 57 & T4NOMO & 103.9 & The middle portion of pons & 2 & $0.4 \times 0.4 \times 0.60 .4 \times 0.5 \times 0.8$ & 2 \\
\hline 3 & female & 37 & T4N2M0 & 102.3 & The middle portion of pons & 1 & $1.0 \times 1.6 \times 2.2$ & 2 \\
\hline 4 & female & 55 & T3N1M0 & 49.3 & The proximal portion of pons & 1 & $1.2 \times 1.4 \times 1.5$ & 4 \\
\hline 5 & male & 53 & T3N3M0 & 124.9 & The proximal portion of pons & 2 & $0.8 \times 0.9 \times 1.30 .4 \times 0.7 \times 1.8$ & 5 \\
\hline 6 & male & 59 & T4N1M0 & 51.8 & The top portion of pons & 1 & $1.3 \times 1.9 \times 0.9$ & 1 \\
\hline 7 & female & 42 & T4N1M0 & 90.2 & The top portion of pons & 1 & $1.2 \times 0.6 \times 1.7$ & 1 \\
\hline 8 & female & 49 & T4N1M0 & 36.5 & The top portion of pons & 1 & $1.0 \times 0.7 \times 0.9$ & 2 \\
\hline
\end{tabular}



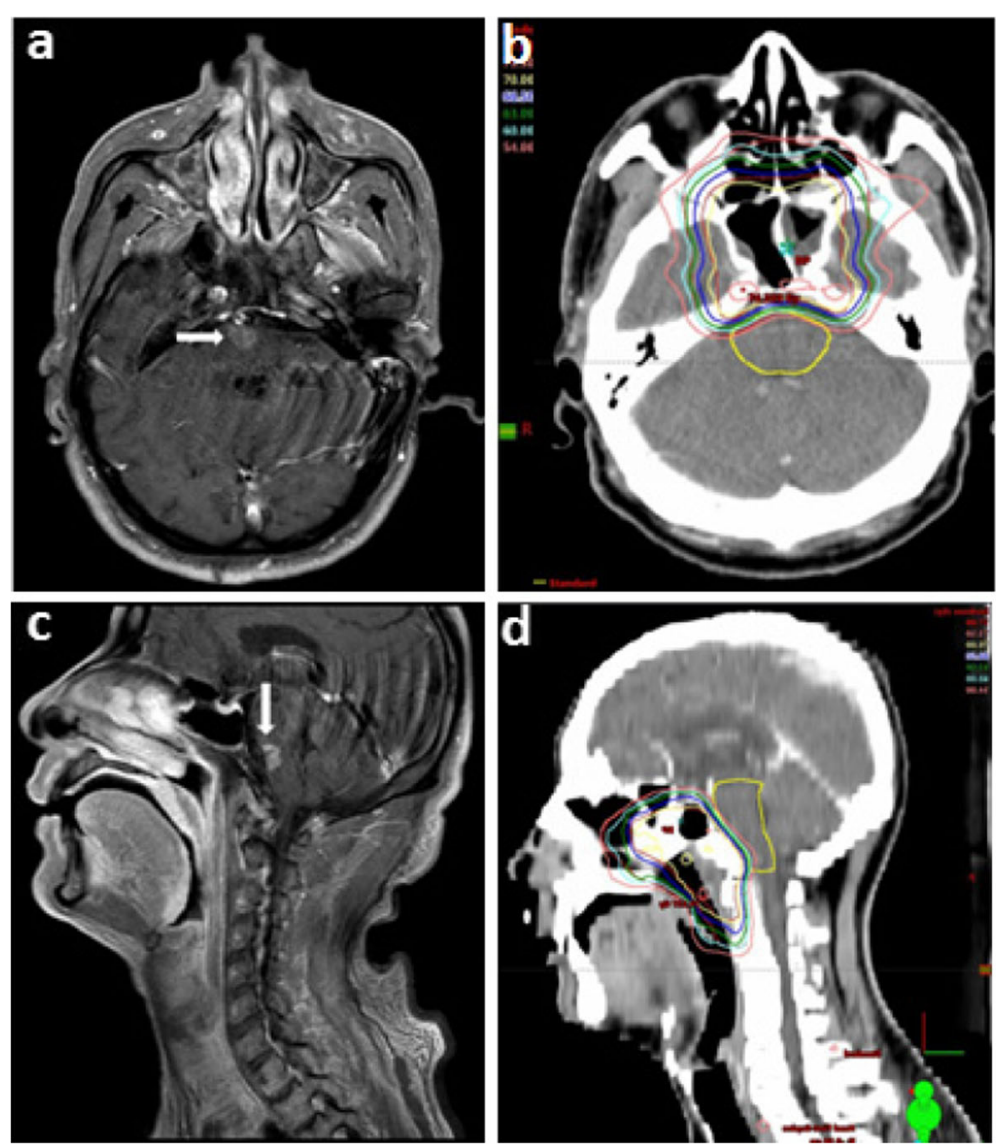

Fig. 2 Necrosis nidus within the brainstem and the corresponding dose distribution. Contrast-enhanced lesion (white arrow) in the axial (a) and sagittal (c) view on post-contrast T1-weighted MRI images with a 53-year-old NPC patient (the 5th patient in Table 1). Corresponding isodose lines are shown in $\mathbf{b}$ and $\mathbf{d}$, respectively

in the anteroposterior diameter of the pons cistern in the enrolled patients, which ranged from $1.9 \mathrm{~mm}$ to $13.8 \mathrm{~mm}$ (median, $4.96 \mathrm{~mm}$ ). However, the Cox regression analysis revealed that it was not a risk factor of radiation-induced brainstem injury.

Table 2 Analysis on radiation dose and other factors affecting brainstem toxicities in Cox regression models

\begin{tabular}{llll}
\hline Factor & $p$-value & HR & $95 \% \mathrm{Cl}$ for HR \\
\hline T category $\left(\mathrm{T}_{4} \mathrm{vs}_{1-3}\right)$ & 0.029 & 5.94 & $1.2-29.43$ \\
GTVnx $\mathrm{D}_{2 \%}$ & 0.040 & 1.21 & $1.01-1.45$ \\
$\mathrm{D}_{\max }$ & 0.003 & 1.15 & $1.05-1.26$ \\
$\mathrm{D}_{1 \%}$ & 0.003 & 1.16 & $1.05-1.28$ \\
$\mathrm{D}_{0.1 \mathrm{ccc}}$ & 0.003 & 1.16 & $1.05-1.27$ \\
$\mathrm{D}_{1 \mathrm{cc}}$ & 0.006 & 1.15 & $1.04-1.27$ \\
\hline
\end{tabular}

$D_{2 \%}$ near the maximum absorbed dose of $\mathrm{GTV}_{\mathrm{nx}}, D_{\max }$ the maximum point dose of brainstem,

$D_{1 \%}$ the dose of $1 \%$ brainstem volume, $D_{0.1 c c}$ the maximum dose of brainstem delivered to a volume of $0.1 \mathrm{ml}, D_{1 c c}$ the maximum dose of brainstem delivered to a volume of $1.0 \mathrm{ml}, \mathrm{GTVnx}$

Gross tumor volume of nasopharyngeal primary lesions and their direct extensions

Abbreviation: $H R$ hazard ratio, $\mathrm{Cl}$ confidence interval, GTV Gross tumor volume
Compared with conventional radiotherapy, IMRT reduced the radiation dose to the brainstem, temporal lobe, and other organs at risk; as a result, the incidence of radiation brainstem injury was significantly decreased. The RTOG study 0225 reported that the $\mathrm{D}_{\max }$ of brainstem in IMRT should not exceed 54 Gy, and for patients with locally advanced NPC, this dose recommendation seems to be conservative.

General studies were undertaken to extract brainstem tolerance data. Brainstem necrosis or MRI-based evidence of injury was reported in some studies [13-17]. Five studies used photons at conventional fractionation $[15,18-21]$ and treatment planning limits on the high-dose component of the brainstem dose including a $\mathrm{V}_{55}<0.1$ cc [18], $\mathrm{D}_{\max }<50$ Gy [19], and $\mathrm{D}_{1 \%} \leq 54$ Gy [21]. Uy et al. [15] reported brainstem necrosis for 1 of 40 meningioma patients treated with serial tomotherapy; $\mathrm{D}_{\max }$ was $55.6 \mathrm{~Gy}$, and the absolute volume of the brain-

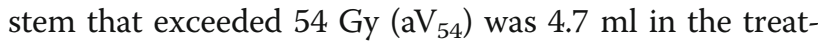
ment plan of this patient. Among 48 patients with NPC treated with $1.2 \mathrm{~Gy} /$ fraction twice daily to $74.4 \mathrm{~Gy}$ and concomitant chemotherapy, Jian noted 3 patients with 
Table 3 Summary of radiation brain stem injury tolerance expressed using ROC curve

\begin{tabular}{lllllllll}
\hline Factor & Area under ROC curve & $\beta$ & $p$ value & Lower limit & Upper limit & Cutoff point & sensitivity & specificity \\
\hline GTVnx & 0.724 & 0.078 & 0.030 & 0.571 & 0.878 & 77.26 & 0.875 & 0.614 \\
$D_{2 \%}$ & & & & & & & \\
$D_{\max }$ & 0.813 & 0.067 & 0.002 & 0.682 & 0.945 & 67.85 & 0.750 & 0.859 \\
$D_{1 \%}$ & 0.818 & 0.068 & 0.002 & 0.685 & 0.952 & 60.75 & 0.875 & 0.803 \\
$D_{0.1 c c}$ & 0.818 & 0.064 & 0.002 & 0.692 & 0.944 & 60.76 & 0.875 & 0.752 \\
$D_{1 \text { cc }}$ & 0.798 & 0.069 & 0.004 & 0.663 & 0.932 & 54.58 & 0.875 & 0.737 \\
\hline
\end{tabular}

$D_{2 \%}$ near the maximum absorbed dose of GTVnx, $D_{\max }$ the maximum point dose of brainstem, $D_{1 \%}$ the dose of $1 \%$ brainstem volume, $D_{0.1 c c}$ the maximum dose of brainstem delivered to a volume of $0.1 \mathrm{ml}, \mathrm{D}_{1 \mathrm{cc}}$ the maximum dose of brainstem delivered to a volume of $1.0 \mathrm{ml}$, GTVnx Gross tumor volume of nasopharyngeal primary lesions and their direct extensions

Abbreviation: ROC Receiver operating characteristic, GTV Gross tumor volume

Grade 1 neurologic deficit [20]. It was also reported in 367 skull-base tumor patients treated with a combination of photon and proton conformal radiation therapy between 1974 and 1995. There were 19 late brainstem-related toxicities, including three deaths. Significant predictors of toxicity by univariate analysis were as follows: $\mathrm{D}_{\max }>64 \mathrm{~Gy}, \mathrm{aV}_{50}>5.9 \mathrm{ml}, \mathrm{aV}_{55}>2.7 \mathrm{ml}$, and $\mathrm{aV}_{60}>0.9 \mathrm{ml}[13,14]$. Of 208 NPC patients with more than 5 years survival after IMRT, one patient $(0.48 \%)$ with a grade 2 brainstem injury and stage $\mathrm{T}_{4}$ (cavernous sinus) was included in the study [22, 23]. For this patient, the highest irradiation dose of the brainstem was 54.54 Gy, with a mean dose of 28.79 Gy. It was shown that the entire brainstem may be treated to 54 Gy using conventional fractionation with acceptable risk of severe or permanent neurological effects. Smaller volumes of the brainstem $(1-10 \mathrm{cc})$ may be irradiated to a maximum dose of 59 Gy with conventional dose fraction (2Gy). The risk appears to increase notably when doses exceed 64 Gy [23]. However, $D_{\max }, D_{1.0 c c}$, and the mean dose of brainstem toxicity outcomes have not been reported in patients with NPC receiving IMRT in long-term follow-up. There is insufficient information to determine whether there is any volume effect.

In this study, brainstem injury lesion occurred most frequently in the proximal or top portion of the pons, extending to the midbrain and medulla oblongata. It may be related to the anatomical structure of the brainstem. The pons easily accepts higher doses; in areas that are located in the posterior closest to the basilar clivus, rather than the other areas, the occurrence of radiation brainstem injury is likely greater. However, lesion sites did not all occur in the most anterior portion of the pons. Four patients' sites were located on both sides of the pons. The dose of the lesion site in 8 cases was less than its $D_{\max }$, suggesting that the occurrence of brainstem injury may be closely related to the brainstem dose per unit volume, so $D_{0.1 c c}$ and $D_{1 \text { cc }}$ were used as indicators to evaluate brainstem injury in this study.

The cutoff point of $D_{\max }$ exceeded the organ at risk (OAR) value limited by RTOG 0225/0615 in our study. There were two explanations for this abnormality. On the one hand, it was difficult to design the radiotherapy plan for the $T_{3}-T_{4}$ stage patients who had the special morphological lesions. On the other hand, insufficient

Table 4 The dose of radiation brain stem injury in 8 patient

\begin{tabular}{|c|c|c|c|c|c|c|c|c|c|c|}
\hline \multirow[t]{2}{*}{ patient } & \multicolumn{4}{|c|}{ GTVnx } & \multirow[t]{2}{*}{$D_{1 \%}$} & \multirow[t]{2}{*}{$D_{\text {mean }}$} & \multirow[t]{2}{*}{$\mathrm{D}_{\max }$} & \multirow[t]{2}{*}{$D_{0.1 c c}$} & \multirow[t]{2}{*}{$D_{1 c c}$} & \multirow[t]{2}{*}{$D_{i}$} \\
\hline & $\mathrm{D}_{2 \%}$ & $\mathrm{BED}_{\mathrm{D} 2 \%}$ & $D_{98 \%}$ & $\mathrm{BED}_{\mathrm{D} 98 \%}$ & & & & & & \\
\hline 1 & 77.69 & 94.16 & 72.10 & 87.39 & 64.35 & 49.64 & 68.47 & 65.60 & 62.11 & 61.30 \\
\hline 2 & 78.60 & 95.26 & 75.01 & 90.91 & 65.20 & 38.04 & 71.59 & 66.96 & 61.46 & 68.60 \\
\hline 3 & 82.32 & 99.77 & 73.52 & 89.11 & 65.10 & 44.99 & 69.72 & 65.93 & 59.03 & 64.30 \\
\hline 4 & 80.66 & 97.76 & 71.23 & 86.33 & 66.44 & 31.75 & 74.07 & 68.64 & 59.25 & 68.20 \\
\hline 5 & 73.49 & 89.07 & 68.90 & 83.51 & 60.15 & 36.43 & 63.38 & 60.89 & 56.57 & 58.10 \\
\hline 6 & 81.79 & 99.13 & 72.70 & 88.11 & 63.73 & 32.40 & 69.41 & 65.79 & 60.00 & 61.73 \\
\hline 7 & 78.80 & 95.51 & 72.06 & 87.34 & 61.80 & 36.66 & 67.87 & 63.60 & 54.60 & 60.00 \\
\hline 8 & 77.32 & 93.71 & 75.25 & 91.20 & 50.94 & 24.85 & 55.30 & 52.47 & 47.77 & 51.71 \\
\hline
\end{tabular}

$D_{2 \%}$ near the maximum absorbed dose of GTVnx, $D_{98 \%}$ near the minimum absorbed dose of GTVnx, $D_{1 \%}$ the dose of $1 \%$ brainstem volume, $D_{\text {mean }}$ the mean dose of brainstem, $D_{\max }$ the maximum point dose of brainstem, $D_{0.1 c c}$ the maximum dose of brainstem delivered to a volume of $0.1 \mathrm{ml}, D_{1 c c}$ the maximum dose of brainstem delivered to a volume of $1.0 \mathrm{ml}, D_{i}$ injury lesion dose of brainstem, GTVnx Gross tumor volume of nasopharyngeal primary lesions and their direct extensions Abbreviation: GTV Gross tumor volume, BED Biological effective dose 


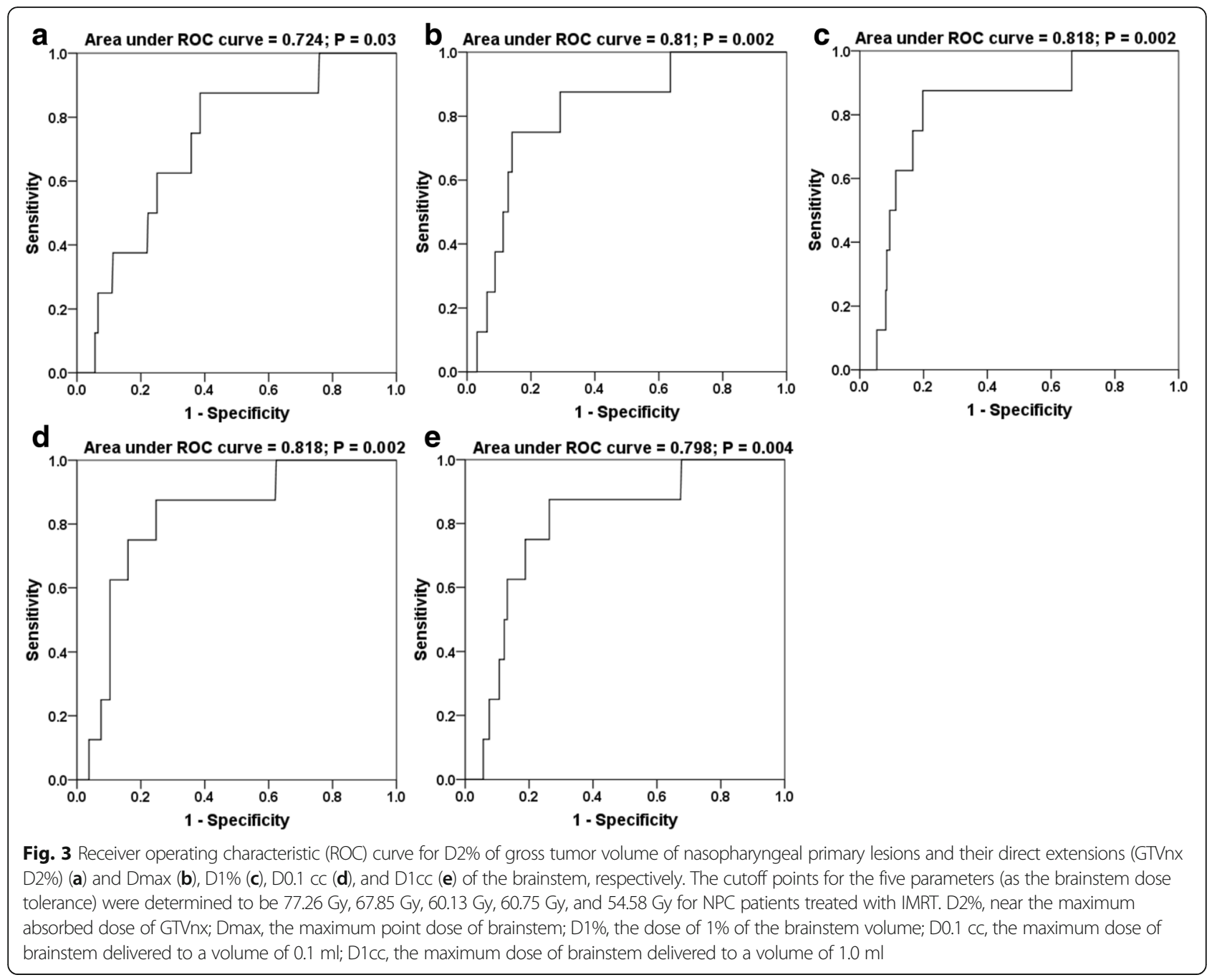

attention was paid to protecting patients' brainstems in the early stage of IMRT. At that time, no uniform standard could be referenced to contour the brainstem, which might cause the brainstem to be exposed to higher doses of radiation.

When reviewing the 8 brainstem injuries, we found two patients with diabetes. We considered their brainstem injury might be related to diabetes because their brainstem doses were not high when compared to other cases. Diabetes will cause microvessel disorder which may aggravate radiation-induced brainstem injury. Thus we reviewed all 327 patients and found that the proportion of diabetes was $12.2 \%$, which was consistent with the most recent national survey in 2010 reported that the rate of diabetes was $11.6 \%$ [24]. Statistical analysis was not possible due to the limited cases. In the fifth and eighth cases with diabetes, the $D_{\max }$ of brainstem injury was lower than the cutoff point. Brainstem-related toxicities may be related to the microcirculation disturbance. GTVnx
$D_{2 \%}, D_{\max }, D_{1 \%}, D_{0.1 c c}$, and $D_{1 c c}$ should be strictly limited in patients with local microcirculation disorders, including diabetes, high blood pressure, immune disturbance, and vascular malformations.

For patients with locally advanced NPC, sometimes it is difficult to balance tumor control and the radiation-induced brainstem injury. In those cases, we hope that the recommended dose-volume parameters are of some assistance. When the brainstem maximum dose was limited, and the unit volume dose was also strictly controlled, radiation-induced brainstem injury was uncommon. More accurate values depend on studies with larger sample sizes and longer follow-up periods.

\section{Conclusions}

In brief, radiation-induced brainstem injury is uncommon in patients with NPC undergoing radiation therapy. Brainstem injury was significantly associated with the radiotherapy dose per unit volume. 


\section{Abbreviations}

ADLs: Activities of daily living; CT: Computed tomography; CTCAE: Common Terminology Criteria for Adverse Events; CTV: Clinical target volume; DVHs: Dose-volume-histograms; GTV: Gross tumor volume; IMRT: Intensity-modulated radiotherapy; MRI: Magnetic resonance imaging;" NPC: Nasopharyngeal carcinoma; OAR: Organ At Risk; QoL: Quality-of-life; RN: Brain necrosis; ROC: Receiver operating characteristic; RTOG: Radiation therapy oncology group

\section{Funding}

This work was supported by grants from Natural Science Foundation of Jiangsu Province of China (SBK2015020256) and General Program of Health Planning Committee of Jiangsu (H201614).

\section{Availability of data and materials}

The datasets used and analyzed during the current study are available from the corresponding author on reasonable request.

\section{Authors' contributions}

CYY, CZZ collected data and drafted the manuscript. JHX and LJW performed the statistical analyses. JJY, LFZ, and GRZ participated in the design of the study. $\mathrm{SFH}$ and $\mathrm{XH}$ conceived of the study and participated in its design. All authors read and approved the final manuscript.

\section{Ethics approval and consent to participate}

All procedures involving human participants were in accordance with the ethical standards of the institutional research committee and with the 1964 Helsinki declaration and its later amendments or comparable ethical standards.

\section{Consent for publication}

All patients gave written informed consent.

\section{Competing interests}

The authors declare that they have no competing interests.

\section{Publisher's Note}

Springer Nature remains neutral with regard to jurisdictional claims in published maps and institutional affiliations.

\section{Author details \\ 'Department of Radiation Oncology, Jiangsu Cancer Hospital \& Jiangsu Institute of Cancer Research \& The Affiliated Cancer Hospital of Nanjing Medical University, 42 Baiziting Road, Nanjing 210009, People's Republic of China. ${ }^{2}$ Department of Medical Oncology, Jiangsu Cancer Hospital \& Jiangsu Institute of Cancer Research \& The Affiliated Cancer Hospital of Nanjing Medical University, 42 Baiziting Road, Nanjing 210009, People's Republic of China. ${ }^{3}$ Department of Imaging, Jiangsu Cancer Hospital \& Jiangsu Institute of Cancer Research \& The Affiliated Cancer Hospital of Nanjing Medical University, 42 Baiziting Road, Nanjing 210009, People's Republic of China.}

Received: 4 July 2018 Accepted: 20 August 2018

Published online: 03 October 2018

\section{References}

1. Yu MC. Diet and nasopharyngeal carcinoma. Prog Clin Biol Res. 1990;346: 93-105.

2. Chan AT. Nasopharyngeal carcinoma. Ann Oncol. 2010;21(Suppl 7):vii308-12. https://doi.org/10.1093/annonc/mdq277.

3. Zhou X, Ou X, Xu T, Wang X, Shen C, Ding J, et al. Effect of dosimetric factors on occurrence and volume of temporal lobe necrosis following intensity modulated radiation therapy for nasopharyngeal. Int J Radiat Oncol Biol Phys. 2014;90(2):261-9. https://doi.org/10.1016/j.jprobp.2014.05.036.

4. Lee N, Harris J, Garden AS, Straube W, Glisson B, Xia P, et al. Intensitymodulated radiation therapy with or without chemotherapy for nasopharyngeal carcinoma: radiation therapy oncology group phase II trial 0225. J Clin Oncol. 2009:27(22):3684-90. https://doi.org/10.1200/JCO. 2008.19.9109.

5. Xu J, He X, Cheng K, Guo W, Bian X, Jiang X, et al. Concurrent chemoradiotherapy with nedaplatin plus paclitaxel or fluorouracil for locoregionally advanced nasopharyngeal carcinoma: survival and toxicity. Head Neck. 2014;36(10):1474-80. https://doi.org/10.1002/hed.23487.
6. Baxi S, Park E, Chong V, Chung HT. Temporal changes in IMRT contouring of organs at risk for nasopharyngeal carcinoma-the learning curve blues and a tool that could help. Technol Cancer Res Treat. 2009; 8(2):131-40. https://doi.org/10.1177/153303460900800206.

7. Greene-Schloesser D, Robbins ME, Peiffer AM, Shaw EG, Wheeler KT, Chan MD. Radiation induced brain injury: a review. Front Oncol. 2012;2:73. https://doi.org/10.3389/fonc.2012.00073.

8. Wang S, Tryggestad E, Zhou T, Armour M, Wen Z, Fu DX, et al. Assessment of MRI parameters as imaging biomarkers for radiation necrosis in the rat brain. Int J Radiat Oncol Biol Phys. 2012;83(3):e431-6. https://doi.org/10. 1016/j.jijobp.2011.12.087.

9. Asai A, Kawamoto K. Radiation-induced brain injury. Brain Nerve. 2008;60(2):123-9.

10. Kased N, Huang K, Nakamura JL, Sahgal A, Larson DA, McDermott MW, et al. Gamma Knife radiosurgery for brainstem metastases: the UCSF experience. J Neurooncol. 2008:86(2):195-205. https://doi.org/10.1007/s11060-007-9458-4.

11. Foote KD, Friedman WA, Buatti JA, Meeks SL, Bova FJ, Kubilis PS. Analysis of risk factors associated with radiosurgery for vestibular schwannoma. J Neurosurg. 2001;95(3):440-9. https://doi.org/10.3171/jns.2001.95.3.0440.

12. Merchant TE, Chitti RM, Li C, Xiong X, Sanford RA, Khan RB. Factors associated with neurological recovery of brainstem function following postoperative conformal radiation therapy in infratentorial ependymoma. Int J Radiat Oncol Biol Phys. 2010;76(2):496-503. https://doi.org/10.1016/j.jirobp. 2009.01.079

13. Debus J, Hug EB, Liebsch NJ, O'Farrel D, Finkelstein D, Efird J, et al. Brainstem tolerance to conformal radiotherapy of skull base tumors. Int J Radiat Oncol Biol Phys. 1997:39(5):967-75.

14. Debus J, Hug EB, Liebsch NJ, O'Farrel D, Finkelstein D, Efird J, et al. Dosevolume tolerance of the brainstem after high-dose radiotherapy. Front Radiat Ther Oncol. 1999;33:305-14.

15. Uy NW, Woo SY, Teh BS, Mai WY, Carpenter LS, Chiu JK, et al. Intensitymodulated radiation therapy (IMRT) for meningioma. Int J Radiat Oncol Biol Phys. 2002;53(5):1265-70.

16. Wenkel E, Thornton AF, Finkelstein D, Adams J, Lyons S, De La Monte S, et al. Benign meningioma: partially resected, biopsied, and recurrent intracranial tumors treated with combined proton and photon radiotherapy. Int J Radiat Oncol Biol Phys. 2000;48(5):1363-70.

17. Clark BG, Souhami L, Pla C, Al-Amro AS, Bahary JP, Villemure JG, et al. The integral biologically effective dose to predict brain stem toxicity of hypofractionated stereotactic radiotherapy. Int J Radiat Oncol Biol Phys. 1998:40(3):667-75

18. Schoenfeld GO, Amdur RJ, Morris CG, Li JG, Hinerman RW, Mendenhall WM Patterns of failure and toxicity after intensity-modulated radiotherapy for head and neck cancer. Int J Radiat Oncol Biol Phys. 2008;71(2):377-85. https://doi.org/10.1016/j.ijrobp.2007.10.010.

19. Hoppe BS, Stegman LD, Zelefsky MJ, Rosenzweig KE, Wolden SL, Patel SG, et al. Treatment of nasal cavity and paranasal sinus cancer with modern radiotherapy techniques in the postoperative setting-the MSKCC experience. Int J Radiat Oncol Biol Phys. 2007;67(3):691-702. https://doi.org/ 10.1016/j.jijrobp.2006.09.023.

20. Jian JJ, Cheng SH, Tsai SY, Yen KC, Chu NM, Chan KY, et al. Improvement of local control of T3 and T4 nasopharyngeal carcinoma by hyperfractionated radiotherapy and concomitant chemotherapy. Int J Radiat Oncol Biol Phys. 2002:53(2):344-52

21. Daly ME, Chen AM, Bucci MK, El-Sayed I, Xia P, Kaplan MJ, et al. Intensitymodulated radiation therapy for malignancies of the nasal cavity and paranasal sinuses. Int J Radiat Oncol Biol Phys. 2007;67(1):151-7. https://doi. org/10.1016/j.ijrobp.2006.07.1389.

22. Zheng Y, Han F, Xiao W, Xiang Y, Lu L, Deng X, et al. Analysis of late toxicity in nasopharyngeal carcinoma patients treated with intensity modulated radiation therapy. Radiat Oncol. 2015;10:17. https://doi.org/10.1186/s13014014-0326-z

23. Mayo C, Yorke E, Merchant TE. Radiation associated brainstem injury. Int J Radiat Oncol Biol Phys. 2010;76(3 Suppl):S36-41. https://doi.org/10.1016/j. ijrobp.2009.08.078.

24. Xu Y, Wang L, He J, Bi Y, Li M, Wang T, et al. Prevalence and control of diabetes in Chinese adults. JAMA. 2013:310(9):948-59. https://doi.org/ 10.1001/jama.2013.168118 